\title{
Pomegranate Fruit and Juice (cv. Mollar), Rich in Ellagitannins and Anthocyanins, Also Provide a Significant Content of a Wide Range of Proanthocyanidins
}

\author{
Huertas M. Díaz-Mula, Francisco A. Tomás-Barberán, ${ }^{*}$ a and Rocío García-Villalba*
}

Laboratory of Food \& Health, Research Group on Quality, Safety and Bioactivity of Plant Foods, Centro de Edafología y Biología Aplicada del Segura-Consejo Superior de Investigaciones Científicas (CEBAS-CSIC), Post Office Box 164, Campus de Espinardo, 30100 Murcia, Spain

\section{Supporting Information}

ABSTRACT: Proanthocyanidins (PAs) were characterized in husk, membranes, arils, and seeds of 'Mollar de Elche' pomegranate cultivar using liquid chromatography-electrospray ionization-ion-trap tandem mass spectrometry after acid catalysis in the presence of phloroglucinol (phloroglucinolysis). PAs were also evaluated in commercial juice and in the juice made in the laboratory by pressing fresh arils. Pomegranate PAs were composed of a very rich mixture of flavan-3-ols: catechin, epicatechin, gallocatechin, epigallocatechin, epigallocatechin gallate, and epicatechin gallate. Catechins and gallocatechins were the most abundant units, while galloylated flavanols were detected at lower concentrations. The highest PA content was found in husk $(1840.5 \mu \mathrm{g} / \mathrm{g})$, followed by seeds $(316.2 \mu \mathrm{g} / \mathrm{g})$ and membranes $(51.4 \mu \mathrm{g} / \mathrm{g})$. With regard to the pomegranate edible parts, arils showed concentrations of $34.4 \mu \mathrm{g} / \mathrm{g}$ and pressed aril juice of $21.3 \mathrm{mg} / \mathrm{L}$, lower than those found in other traditional PA-containing food and beverages, such as apple, grapes, chocolate, red wine, or tea. Higher concentrations similar to those found in red wine were observed in commercial whole fruit pressed pomegranate juice $(146.9 \mathrm{mg} / \mathrm{L})$. These PA concentrations represented between 0.1 and $7 \%$ of the total polyphenol content in the different pomegranate samples, which were richer in ellagitannins and anthocyanins. The mean degree of polymerization (1.2-2.1) revealed that PAs in Mollar pomegranate samples are constituted mainly of monomers and dimers, which may influence in better absorption and bioavailability of these compounds.

KEYWORDS: pomegranate, proanthocyanidins, phloroglucinolysis, ellagitannins, anthocyanins

\section{INTRODUCTION}

Pomegranate (Punica granatum L.) is one of the oldest known edible fruit widely consumed as fresh fruit and juice. This fruit has generated great interest because its consumption has been associated with positive effects on human health ${ }^{1,2}$ that have been attributed to its high content in phytochemicals, mainly polyphenols. ${ }^{3}$ Most studies on pomegranate polyphenols have focused on two of the major classes of compounds: hydrolyzable tannins (ellagitannins) and anthocyanins. ${ }^{4-7}$ More recently, a large number of colored anthocyanin-flavanol and flavanolanthocyanin adducts have been reported in pomegranate, although at very low concentrations. ${ }^{5,8}$ However, few data are available on other phenolic compounds, such as proanthocyanidins (PAs).

PAs, also known as condensed tannins, are a group of oligomeric or polymeric flavan-3-ols widely distributed in the plant kingdom, mainly in fruit and tree nuts. ${ }^{9}$ PAs containing catechin (C) or epicatechin (EC) are named procyanidins; those containing gallocatechin (GC) or epigallocatechin (EGC) are called prodelphinidins; and those with afzelechin or epiafzelechin are propelargonidins. ${ }^{10}$ Their potent in vitro antioxidant capacity suggested a protective effect for the prevention of diseases related to oxidative stress, such as cardiovascular diseases, type 2 diabetes, and cancer. ${ }^{10,11}$ Their separation and identification is often very difficult as a result of their polymeric nature, large structural diversity, and their presence in complex matrixes. ${ }^{12}$ The molar absorbance of PAs is also lower than those of other phenolic compounds, and this complicates their quantification even more using ultraviolet (UV) detectors. Different methodologies have been developed for the analysis of PAs that include spectrophotometric methods, normal-phase high-performance liquid chromatography (HPLC) coupled to fluorescence detection, derivatization with different reagents, and depolymerization reactions using toluene- $\alpha$-thiol and phloroglucinol. ${ }^{12}$ Phloroglucinolysis is the depolymerization of PAs in an acid environment in the presence of phloroglucinol and gives access to important information regarding PA composition. ${ }^{13}$ This methodology has been widely used in the literature for the characterization of PAs in different foods, including wine, apple, grapes, tea, cocoa, legume seeds, and strawberries. ${ }^{14-16}$

In the case of pomegranate, little interest in PAs has been evidenced thus far. Total PAs have been estimated in pomegranate by spectrophotometric methods, ${ }^{17}$ which are generally non-specific and do not allow for the knowledge of the content of individual compounds. In other publications, the

Special Issue: Health-Promoting Food Ingredients

Received: December 21, 2018

Revised: February 13, 2019

Accepted: February 15, 2019

Published: February 15, 2019 


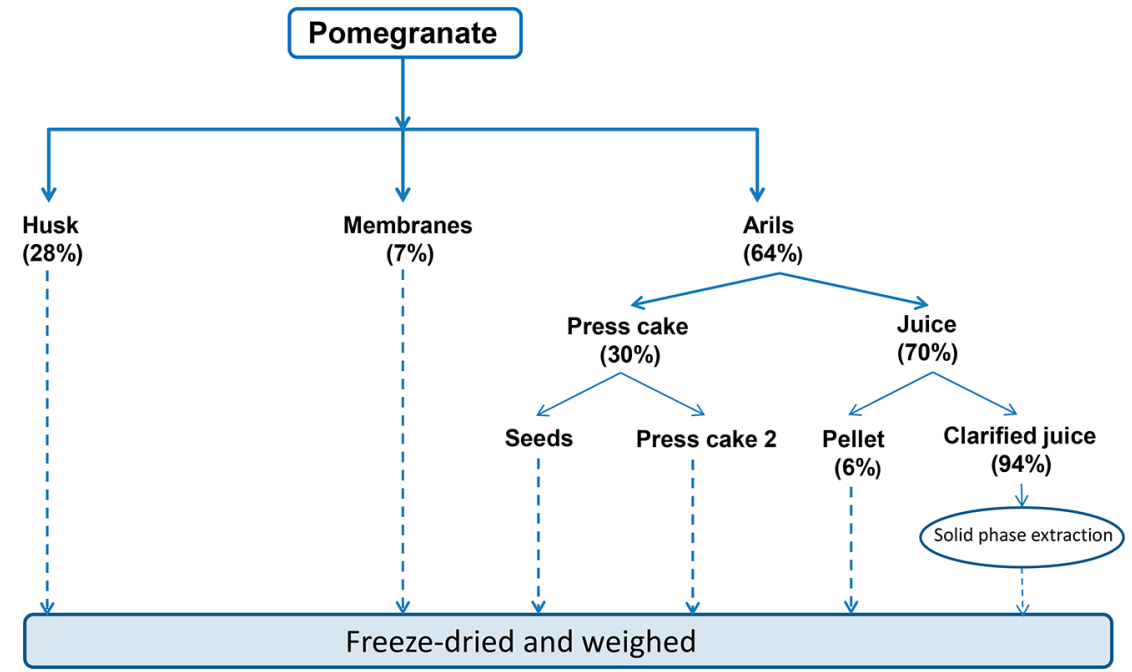

Figure 1. Scheme of the separation procedure of the different parts of pomegranate fruit to be analyzed. In parentheses, the percentage of each part is indicated.

occurrence of flavanols of a low degree of polymerization (mainly monomers and dimers) were reported in pomegranate samples. ${ }^{18,19}$ In particular, the presence of (epi)-catechin, (epi)gallocatechin, and different PA dimers in pomegranate fruit has been described. ${ }^{18}$ In other studies, gallocatechins and a range of prodelphinidins were purified by HPLC from pomegranate peel. $^{20}$ However, knowledge of the occurrence of PAs in pomegranates is still fairly poor, and detailed quantitative information on the PA profile is lacking. The available reports do not provide data on PA subunit composition or the degree of polymerization as well as the content of PAs in the edible parts of pomegranates and the juices prepared with this fruit.

The aim of this work was to study the PA profile (subunit composition, quantitation, and degree of polymerization) of different parts of pomegranate fruit and juices using phloroglucinolysis combined with analysis by liquid chromatography-electrospray ionization-tandem mass spectrometry (LC-ESI-MS/MS).

\section{MATERIALS AND METHODS}

Chemicals. Phloroglucinol and standards of proanthocyanidin B2, (+)-catechin (C), (-)-epicatechin (EC), (-)-gallocatechin (GC), $(-)$-epigallocatechin (EG), (-)-gallocatechin gallate $(\mathrm{GG}),(-)$-epigallocatechin gallate (EGCG), (-)-catechin gallate (CG), (-)-epicatechin gallate (ECG), ellagic acid, and cyanidin 3-O-glucoside were purchased from Sigma-Aldrich (St. Louis, MO, U.S.A.). Stock solutions for each compound at a concentration of $2000 \mathrm{mg} / \mathrm{L}$ were first prepared in methanol, except for ellagic acid that was prepared in dimethyl sulfoxide (DMSO), and then serially diluted to working concentrations. Acetonitrile and methanol of LC-MS grade were from J.T. Baker (Deventer, Netherlands), DMSO was from LabScan (Dublin, Ireland) and formic acid, hydrochloric acid, and sodium acetate were from Panreac (Barcelona, Spain). Ascorbic acid was from Acros Organics (Geel, Belgium). All other chemicals and reagents were of analytical grade. Milli-Q system (Millipore Corp., Bedford, MA, U.S.A.) ultrapure water was used throughout the study.

Pomegranate Samples. Pomegranates (4 samples) of cultivar Mollar Elche (Elche, Spain) harvested in October 2016 were obtained from a local market. The fruit was manually separated into husk, membranes, and arils (Figure 1). Husk and membranes were weighed, freeze-dried, and ground with a mixer grinder (Oster Professional BPST02-B), and the powders kept in a desiccator before the analyses. Arils were not able to be freeze-dried and were analyzed directly because their high sugar content prevented an effective freeze drying.
The arils were pressed with a hand-press juicer to obtain the press cake and the juice, which was subsequently filtered through cheesecloth to remove solid particles. Seeds were separated from the press cake remaining after pressing the arils. The seeds, press cake, and pellet obtained after filtration of the juice were freeze-dried and ground with the mixer grinder. Clarified juice was analyzed directly for the determination of anthocyanins and ellagitannins but was passed through Sep-Pak cartridges before the phloroglucinolysis reaction. Commercial pomegranate juices (also from 'Mollar' cultivar), obtained by pressure extraction of the whole fruit, were provided by a juice processor (AMC, Murcia, Spain). Juices were centrifuged at $3500 \mathrm{~g}$ for $10 \mathrm{~min}$, and the pellet obtained was freeze-dried and ground. The moisture content was determined from the weight of the samples before and after freeze drying.

Analysis of Proanthocyanidins by Phloroglucinolysis. Sample Preparation. Solid samples (husk, membranes, seeds, press cake, and pellets) were processed directly after freeze drying. Pomegranate juice samples (both made in the laboratory from the arils and commercial samples) were filtered through reversed-phase solid-phase extraction (SPE) cartridges before the hydrolysis reaction to remove sugar and organic acids and, thus, facilitate the freeze drying of the samples. A total of $20 \mathrm{~mL}$ of samples was loaded onto a SPE cartridge Chromafix C18 (950 mg, Machery-Nagel, Düren, Germany), which was previously conditioned with $10 \mathrm{~mL}$ of $\mathrm{MeOH}$ and then $10 \mathrm{~mL}$ of water. After sample filtration, the cartridge was washed with $10 \mathrm{~mL}$ of water and the phenolic compounds eluted with $1 \mathrm{~mL}$ of methanol. The methanol extract was evaporated in a speed-vacuum concentrator under reduced pressure, dissolved in $1 \mathrm{~mL}$ of water, freezed at $-80^{\circ} \mathrm{C}$, and freeze-dried. To obtain enough sample quantity, this protocol was repeated 4 times per sample and the powder obtained was collected and weighed.

Phloroglucinolysis Reaction and Analysis by LC-ESI-MS. PAs were quantified as previously reported by Kennedy and Jones, ${ }^{13}$ with some modifications. ${ }^{21}$ A solution of $0.1 \mathrm{M} \mathrm{HCl}$ in methanol containing $50 \mathrm{~g} / \mathrm{L}$ phloroglucinol and $10 \mathrm{~g} / \mathrm{L}$ ascorbic acid was prepared. A total of $50 \mathrm{mg}$ of dried samples was mixed with the phloroglucinolysis reagent $(0.8 \mathrm{~mL})$ and incubated in a water bath for $20 \mathrm{~min}$ at $50{ }^{\circ} \mathrm{C}$. The reaction was stopped by placing the vials in ice and adding $1 \mathrm{~mL}$ of 40 $\mathrm{mM}$ sodium acetate. The samples were centrifuged at $3500 \mathrm{~g}$ for $10 \mathrm{~min}$ and filtered with a $0.22 \mu \mathrm{m}$ polyvinylidene fluoride (PVDF) filter prior to the injection. The same reaction was applied to $1 \mathrm{mg}$ of proanthocyanidin B2 using proportional amounts of reagents. Samples were analyzed using an Agilent HPLC 1200 series instrument equipped with a diode array detector (Agilent Technologies, Waldbronn, Germany) and an ion-trap mass spectrometer detector in series (Bruker Daltonics, Bremen, Germany). Separation of phenolic compounds was achieved on a reverse-phase C18 Pursuit XRs column 
$(250 \times 4 \mathrm{~mm}, 5 \mu \mathrm{m}$ particle size, Agilent Technologies, Waldbronn, Germany), operating at room temperature and a flow rate of $0.8 \mathrm{~mL} /$ min. A total of $10 \mu \mathrm{L}$ of sample was injected. The mobile phases were water with acetic acid $(2.5 \%)$ (phase A) and acetonitrile (phase B). The gradient changed according to the following conditions: $0 \mathrm{~min}, 3 \% \mathrm{~B}$, from 0 to $5 \mathrm{~min}, 3-9 \% \mathrm{~B}$; from 5 to $15 \mathrm{~min}, 9-16 \% \mathrm{~B}$; from 15 to 45 min, $16-50 \%$ B; from 45 to $47 \mathrm{~min}, 50-90 \% \mathrm{~B}$ and maintained at $90 \%$ $\mathrm{B}$ for $1 \mathrm{~min}$, then decreased to the initial conditions in $3 \mathrm{~min}$, and maintained for $5 \mathrm{~min}$ (total time of $57 \mathrm{~min}$ ). The ultraviolet-visible (UV-vis) chromatograms were recorded at $280 \mathrm{~nm}$. The optimal conditions of the electrospray interface were as follows: gas temperature, $325{ }^{\circ} \mathrm{C}$; drying gas, $11 \mathrm{~L} / \mathrm{min}$; and nebulizer pressure, 65 psi. Spectra were acquired in the range of $m / z 50-800$ (target mass of 400) in the negative ionization mode. Compound stability was set at $75 \%$. Flavan-3-ol monomers and phloroglucinol adducts obtained after the phloroglucinolysis reaction were identified using their elution order, absorbance spectra, and mass fragmentation patterns (MS/MS) compared to available standards when possible. Quantification was developed in MS, using the area of the extracted ion chromatograms (EICs) for each compound. Flavan-3-ol monomers were quantified with their own commercial standards, and phloroglucinol adducts were quantified with their corresponding free flavan-3-ol, assuming higher response in MS for adducts (twice more, as indicated below from the phoroglucinolysis of proanthocyanidin B2). The concentrations of flavan-3-ols in the products analyzed were expressed in micrograms per gram of fresh weight (FW) in solid parts and milligrams per liter in juices, taken into account the moisture of the samples. To calculate the apparent mean degree of polymerization $(\mathrm{mDP})$, the sum of all subunits (flavan-3-ol monomer and phloroglucinol adducts, in moles) was divided by the sum of all flavan-3-ol monomers (in moles).

Analysis of Phenolic Profile by Liquid ChromatographyElectrospray Ionization-Ion-Trap Mass Spectrometry (LCESI-IT MS). For the analysis of the phenolic profile, $150 \mathrm{mg}$ of freeze-dried samples (husk, membranes, seeds, press cake, and pellets) were weighted and extracted with $10 \mathrm{~mL}$ of $\mathrm{MeOH} / \mathrm{DMSO} /$ water (40:40:20) acidified with $0.1 \% \mathrm{HCl}$. The sample was then vortexed for $30 \mathrm{~s}$, followed by sonication in an ultrasonic bath for $10 \mathrm{~min}$. The sample was then centrifuged at $3500 \mathrm{~g}$ for $10 \mathrm{~min}$, and the supernatant was filtered through a $0.22 \mu \mathrm{m}$ PVDF filter previous to the injection into the LC-ESI-MS system. Pomegranate juice samples were centrifuged at $3500 \mathrm{~g}$ for $10 \mathrm{~min}$, and the supernatant was filtered through a $0.22 \mu \mathrm{m}$ PVDF filter and directly injected into the LC-ESI-MS system. These samples were analyzed with the same high-performance liquid chromatography-diode array detection-ion-trap mass spectrometry (HPLC-DAD-IT MS) equipment described above for the analysis of the products of phloroglucinolysis and with the same chromatographic column. The chromatographic and MS conditions were similar to those described in previous studies for the analysis of phenolic compounds. ${ }^{21}$ A qualitative approach was applied for the identification of monomers and oligomers (dimers, trimers, tetramers, etc.) of flavan-3-ols. Anthocyanins were quantified in UV at $520 \mathrm{~nm}$ using a calibration curve of cyanidin-3-glucoside. The main ellagitannins were quantified at $360 \mathrm{~nm}$, using a calibration curve of punicalagin for the quantification of punicalagin and punicalin and ellagic acid for the quantification of ellagic acid and its conjugates.

Analysis of Ellagitannins after Acid Hydrolysis by LC-ESIMS. To obtain more accurate quantification of total ellagitannins, an acid hydrolysis of pomegranate samples was applied using a method previously reported. ${ }^{22}$ Briefly, $50 \mathrm{mg}$ of freeze-dried pomegranate samples or $3.34 \mathrm{~mL}$ of juice was directly hydrolyzed with $4 \mathrm{M} \mathrm{HCl}$ in water at $90{ }^{\circ} \mathrm{C}$ for $24 \mathrm{~h}$. After cooling to room temperature and adjusting the $\mathrm{pH}$ to 2.5 , the samples were centrifuged for $10 \mathrm{~min}$ at $3500 \mathrm{~g}$. The supernatant was recovered, and the volume was adjusted to $10 \mathrm{~mL}$ with water and was filtered through a $0.45 \mu \mathrm{m}$ PVDF filter before injection onto the HPLC column. The resulting pellets were dissolved into $10 \mathrm{~mL}$ of $\mathrm{DMSO} / \mathrm{MeOH}(50: 50, \mathrm{v} / \mathrm{v})$, and after centrifugation, the supernatant was also filtered before the injection. Both extracts were analyzed on the same HPLC-DAD-ESI-IT described above with the same instrumental parameters previously reported. ${ }^{22}$ The method was based on the quantification of the acid hydrolysis products that included ellagic acid, gallic acid, sanguisorbic acid dilactone, valoneic acid dilactone, and gallagic acid dilactone. All of the hydrolysis products were quantified with the calibration curve of ellagic acid at $360 \mathrm{~nm}$, except gallic acid that was quantified with an authentic standard at 280 $\mathrm{nm}$.

\section{RESULTS AND DISCUSSION}

Qualitative Identification of PAs by LC-ESI-MS. The PA profile in pomegranate samples was studied using the general method for polyphenol analysis. The detection with UV detectors was difficult as a result of their low concentration and the high complexity of the samples rich in anthocyanidins and ellagitannins. Masses of PA monomers, oligomers, and polymers were extracted from the MS chromatogram. Monomers $(\mathrm{m} / z 289$ and 305) and dimers $(\mathrm{m} / z$ 577, 609, and 593) of catechins and/or gallocatechins were detected with high intensity (Figure 2), and the presence of different isomers

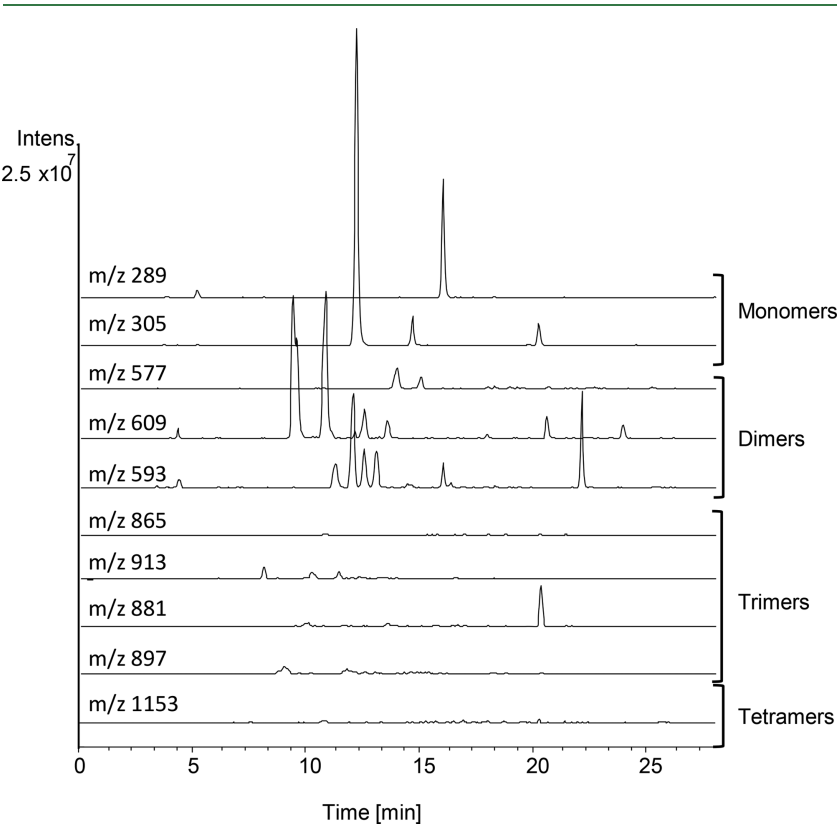

Figure 2. EIC of the main monomers, dimers, trimers, and tetramers searched in the pomegranate samples: $m / z 289$, (epi)catechin; $m / z$ 305, (epi)gallocatechin; $m / z$ 577, (epi)catechin-(epi)catechin; $m / z$ 609, (epi)gallocatechin-(epi)gallocatechin; $m / z$ 593, (epi)gallocatechin-(epi)catechin; $m / z$ 865, (epi)catechin-(epi)catechin(epi)catechin; $m / z$ 913, (epi)gallocatechin-(epi)gallocatechin-(epi)gallocatechin; $m / z$ 881, (epi)gallocatechin-(epi)catechin-(epi)catechin; $m / z$ 897, (epi)gallocatechin-(epi)gallocatechin-(epi)catechin; and $m / z$ 1153, (epi)catechin-(epi)catechin-(epi)catechin-(epi)catechin.

was observed. The same monomers and dimers where previously detected in pomegranate samples. ${ }^{18}$ Some trimers composed of catechins and gallocatechins $(\mathrm{m} / z 881$ and 913) with masses that could also correspond to dimers of ECG and EGCG were identified but with much lower intensities. Masses of other trimers, tetramers, and higher polymers were not detected. This could be due to (1) the absence of these polymers in pomegranate samples, (2) their presence at undetectable concentrations, or (3) that a significant amount of polymeric PAs remains in the residue after extraction. Quantification was difficult due to the lack of available standards and the possible co-elution of the oligomers as a large unresolved peak. Acid- 
Table 1. Proanthocyanidin Cleavage Products Found in Pomegranate Samples after Acid Catalysis in the Presence of Phloroglucinol

\begin{tabular}{|c|c|c|c|c|c|c|}
\hline compound & number $^{a}$ & & Rt (min) & {$[\mathrm{M}-\mathrm{H}]^{-}$} & $\lambda_{\max }$ & MS/MS \\
\hline \multirow[t]{2}{*}{$(-)$-gallocatechin } & 1 & adduct & 5.6 & 429 & 278 & $303,261,177$, and 125 \\
\hline & 3 & monomer & 7.9 & 305 & 278 & $287,261,219$, and 179 \\
\hline \multirow[t]{2}{*}{ (-)-epigallocatechin } & 2 & adduct & 6.2 & 429 & 271 & $303,261,177$, and 125 \\
\hline & 7 & monomer & 11.6 & 305 & 271 & $287,261,219$, and 179 \\
\hline \multirow[t]{2}{*}{$(+)$-catechin } & 4 & adduct & 9.1 & 413 & 278 & $287,261,175$, and 125 \\
\hline & 8 & monomer & 13.0 & 289 & 278 & $271,245,205$, and 179 \\
\hline \multirow[t]{2}{*}{ (-)-epigallocatechin gallate } & 5 & adduct & 9.4 & 581 & 275 & $537,455,429,261$, and 175 \\
\hline & 10 & monomer & 16.8 & 457 & 277 & $413,331,305$, and 169 \\
\hline \multirow[t]{2}{*}{$(-)$-epicatechin } & 6 & adduct & 9.8 & 413 & 278 & $287,261,175$, and 125 \\
\hline & 11 & monomer & 17.1 & 289 & 278 & $271,245,205$, and 179 \\
\hline \multirow[t]{2}{*}{ (-)-epicatechin gallate } & 9 & adduct & 15.2 & 565 & 277 & $439,413,395,261$, and 175 \\
\hline & 12 & monomer & 23.2 & 441 & 273 & $397,331,289,193$, and 169 \\
\hline
\end{tabular}

${ }^{a}$ Numbers correspond to peaks in Figure 4.<smiles>[R]O[C@H]1Cc2c(O)cc(O)cc2O[C@H]1c1cc([R])c(O)c(O)c1</smiles>

$$
\begin{array}{ll}
\mathrm{R}=\mathrm{H} & \text { catechin } \\
\mathrm{R}=\mathrm{OH} & \text { gallocatechin }
\end{array}
$$

$\mathrm{R}=\mathrm{H} \quad \mathrm{R}^{\prime}=\mathrm{H} \quad$ epicatechin

$\mathrm{R}=\mathrm{H} \quad \mathrm{R}^{\prime}=$ gallate epicatechin gallate

$\mathrm{R}=\mathrm{OH} \mathrm{R}^{\prime}=\mathrm{H} \quad$ epigallocatechin

$\mathrm{R}=\mathrm{OH} \mathrm{R}^{\prime}=$ gallate epigallocatechin gallate

Figure 3. Basic structures of flavan 3-ols in pomegranate.

catalyzed cleavage in the presence of phloroglucinol was applied for an accurate quantification of PAs.

Phloroglucinolysis Reaction and Method Validation. A variety of flavan-3-ol monomers and their phloroglucinol adducts were detected in pomegranate samples after the phloroglucinolysis degradation (Table 1 and Figure 3). All showed similar spectra with maxima around $275 \mathrm{~nm}$. The fragmentation profile was similar for all adducts, with losses of $\mathrm{m} / z 126$ and fragments with $\mathrm{m} / z 125$ corresponding to a phloroglucinol residue. Other fragments at $\mathrm{m} / z 261$ and 177 or 175 were also common in adducts. The monomers, C, EC, GC, and EGC, showed similar fragmentation profiles with losses of $m / z 18$ and 44 and a common fragment at $m / z 179$. EGCG and ECG showed losses of $\mathrm{m} / z 44$ and 152 and fragments at $\mathrm{m} / \mathrm{z}$ 169 , corresponding to the gallic acid residue.

As a result of the small amount of these compounds in the pomegranate samples, their low UV molar absorptivity at 280 $\mathrm{nm}$ and the interference caused by the presence of high quantities of anthocyanins and ellagitannins, the quantitation using an UV detector, as it had been performed thus far, ${ }^{14,21}$ was not possible. A much better response was obtained with the MS detector using EICs of each compound (Figure 4). Flavan-3-ol monomers were quantified with their own available standards, and the method was validated in terms of linearity, limit of detection (LOD) and limit of quantification (LOQ), and precision (intra- and interday) (Table 2). All calibration curves were linear until $100 \mu \mathrm{M}$, and the LOQ was between $0.8 \mu \mathrm{M}$ for $\mathrm{EC}$ and $7.3 \mu \mathrm{M}$ for EGC. Intra- and interday precisions were in all cases below 5 and $15 \%$, respectively. Relative response factors

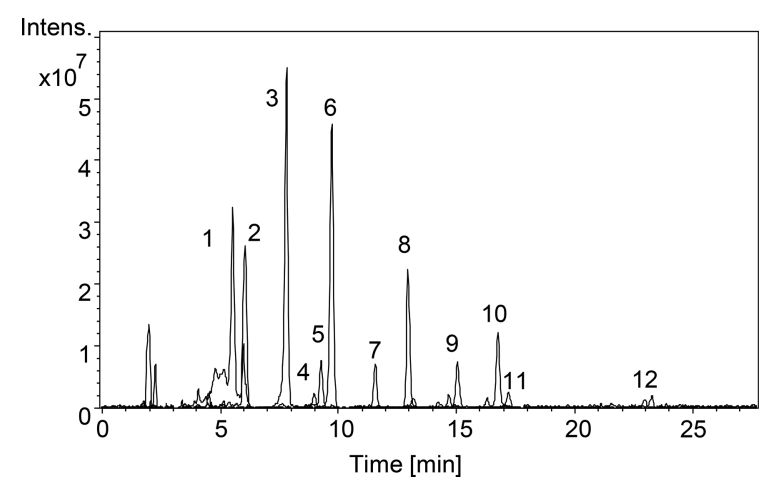

Figure 4. EICs of the flavan-3-ols and their adducts quantified in samples of pomegranate husk after phloroglucynolisis: (1) gallocatechin adduct, (2) epigallocatechin adduct, (3) gallocatechin, (4) catechin adduct, (5) epigallocatechin gallate adduct, (6) epicatechin adduct, (7) epigallocatechin, (8) catechin, (9) epicatechin gallate adduct, (10) epigallocatechin gallate, (11) epicatechin, and (12) epicatechin gallate.

(RRFs) with respect to $\mathrm{C}$ (the more available standard) showed similar responses for GC (0.81), ECG (1.0), and EC (1.37) and higher response in MS for EGCG (2.22), GCG (2.04), ECG (3.50), and CG (3.0). This information confirmed the different response in MS of compounds with a similar structure and the need of using authentic standards when MS is used to quantify. As a result of the absence of available standards for phloroglucinol adducts, phloroglucinolysis of proanthocyanidin 
Table 2. Evaluation of the Linearity, RRF, LOD and LOQ and Precision (Intra- and Interday) for the Analysis of Flava-3-ols in LC-ESI-MS

\begin{tabular}{|c|c|c|c|c|c|c|c|}
\hline & Rt (min) & linearity $(\mu \mathrm{M})$ & $\mathrm{RRF}^{a}(\mathrm{MS})$ & $\mathrm{LOD}(\mu \mathrm{M})$ & $\operatorname{LOQ}(\mu \mathrm{M})$ & intraday precision (\%) & interday precision (\%) \\
\hline$(-)$-gallocatechin & 7.9 & LOD-100 & 0.81 & 2.2 & 7.3 & 3.5 & 11.6 \\
\hline (-)-epigallocatechin & 11.6 & LOD-100 & 1.00 & 1.6 & 5.4 & 2.8 & 12.2 \\
\hline$(+)$-catechin & 13.0 & LOD-100 & 1.00 & 0.6 & 2.0 & 4.3 & 9.7 \\
\hline (-)-epigallocatechin gallate & 16.8 & LOD-100 & 2.22 & 0.7 & 2.3 & 2.4 & 9.1 \\
\hline$(-)$-epicatechin & 17.1 & LOD-100 & 1.37 & 0.2 & 0.8 & 2.2 & 12.0 \\
\hline$(-)$-gallocatechin gallate & 18.4 & LOD -100 & 2.04 & 0.6 & 2.0 & 3.8 & 13.6 \\
\hline$(-)$-epicatechin gallate & 23.2 & LOD -100 & 3.50 & 0.5 & 1.7 & 4.0 & 9.6 \\
\hline$(-)$-catechin gallate & 23.6 & LOD-100 & 3.00 & 0.4 & 1.2 & 5.1 & 11.8 \\
\hline
\end{tabular}

B2 was carried out to study the relative response of the adduct of EC with respect to its monomer. The response in MS of the adduct was 2.5 times more intense than that observed for free EC (Figure 5). Therefore, the same behavior was assumed for

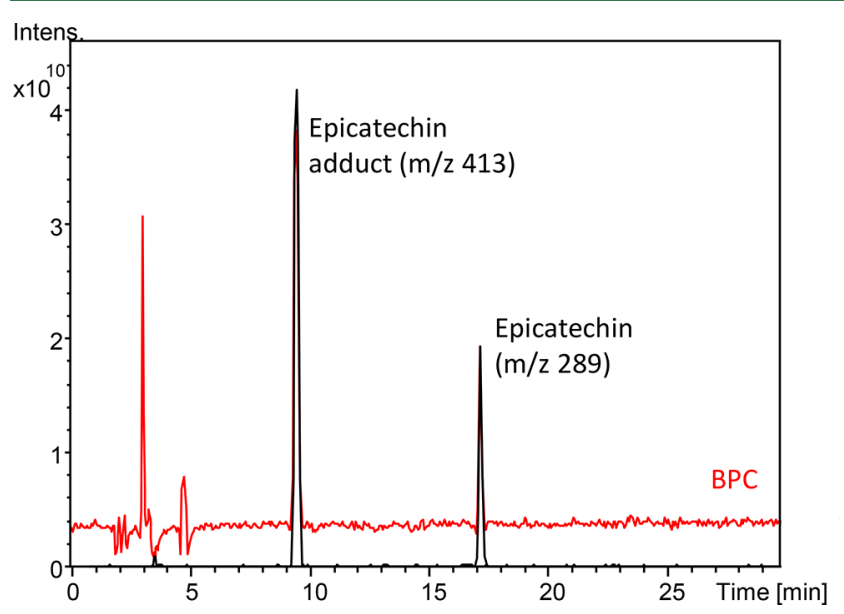

Figure 5. Base peak chromatogram (BPC) (red) and EICs (black) of the compounds obtained after phloroglucinolysis of proanthocyanidin B2.

the rest of the adducts, and a response factor of 2.5 was applied for the quantification of these compounds. This is a good estimation that provides valuable information about the quantity (at least on the order of magnitude).

Proanthocyanidin Composition. Flavan-3-ol monomers and their phloroglucinol adducts were quantified in different parts of the pomegranate fruit and juices (pressed aril juice and commercial juice) from the Mollar cultivar (Table 3). From a qualitative point of view, pomegranate samples showed a very rich profile of $\mathrm{PAs}$ with a variety of monomeric flavan-3-ol units present in their molecules (C, EC, GC, EGC, ECG, and EGCG). Similar qualitative profiles have been described in green tea, ${ }^{23}$ Quercus leaf infusions, ${ }^{24}$ or red wine. ${ }^{25}$ Previous studies reporting concentrations of flavan-3-ols in common Spanish ${ }^{18}$ and American ${ }^{26}$ food products showed that, in general, catechins ( $\mathrm{C}$ and $\mathrm{EC}$ ) are found in all of the flavanol-containing products but the presence of gallocatechins (GC and EGC) is only relevant in some products, including broad bean, lentil, grape, wine, beer, coffee, tea, and most of the berries. Galloyled flavanols (ECG and EGCG) are still less common, and their presence was important only in green and black tea, strawberry, and grape.

Pomegranate husk contained the largest PA content (1840 $\mu \mathrm{g} / \mathrm{g})$ compared to seeds $(316.2 \mu \mathrm{g} / \mathrm{g})$ and membranes $(51.4$ $\mu \mathrm{g} / \mathrm{g})$. The amount in the arils, the edible part of pomegranate fruit, was even lower $(34.4 \mu \mathrm{g} / \mathrm{g})$. The predominant flavan-3-ol units in husk and membrane were GC (51 and 41\%, respectively) and $C$ (15 and $43 \%$, respectively), whereas in seeds and arils, C (55 and 38\%, respectively) and EC (30 and $32 \%$, respectively) were the most abundant units. The presence of gallocatechins and prodelphinidins in pomegranate peel was previously reported. ${ }^{20}$ In the case of juices, the amount in commercial juice $(146.9 \mathrm{mg} / \mathrm{L})$ was much higher than in pressed aril juice $(21.3 \mathrm{mg} / \mathrm{L})$, probably because, in commercial juice, the whole fruit is pressed and the contact with husk and membranes could enrich the juice in these compounds. Different flavan-3-ol units were present in both juices with high percentages of EC and GC. In all pomegranate samples, EGCG and ECG were present but at low concentrations (less than $8 \%$ ). It was difficult to properly compare these results to those found in the literature as a result of scarce information available about the PA content in pomegranate samples and the different methodologies used to quantify them.

In the literature, only one study investigating phloroglucinolysis of PAs in different pomegranate juices was found. ${ }^{27}$ However, in this study, only C (112-148 mg/L) and the phloroglucinol product of C $(66.8-116 \mathrm{mg}$ of catechin equivalent/L) were identified, while no other type of flavan-3ol was detected. In another study, gallocatechins and a range of prodelphinidins were purified by HPLC from pomegranate peel. Gallocatechin, gallocatechin-(4-8)-catechin, gallocatechin-(4$8)$-gallocatechin, and catechin-(4-8)-gallocatechin were all identified, purified, and quantified. ${ }^{19,20}$ The amount of monomers and dimers were quantified in arils, reporting a total amount of $10.9 \mu \mathrm{g} / \mathrm{g} .{ }^{18}$ The amounts found were so low that some authors even reported that flavan-3-ol monomers and procyanidin dimers and trimers were components not usually detected in $100 \%$ pomegranate beverages. ${ }^{28}$

In comparison of the results to commonly consumed foods rich in PAs (apples, green tea, cocoa, red wine, grapes, and berries), the amount found in the pomegranate fruit edible part was low. Some varieties of apples showed higher concentrations of PAs (between 800 and $1410 \mu \mathrm{g} / \mathrm{g})^{26}$ and between 1703 and $5329 \mu \mathrm{g} / \mathrm{g}$ when phloroglucynolusis was used for the quantification. ${ }^{21}$ Chocolate showed the concentration around $5000 \mu \mathrm{g} / \mathrm{g},{ }^{29}$ and grape seeds, also quantified after the pholoroglucinolysis reaction, showed the concentration around $2395 \mu \mathrm{g} / \mathrm{g} .{ }^{30}$ Plums showed concentrations of $2159 \mu \mathrm{g} / \mathrm{g},{ }^{26}$ and berries showed the concentration between 230 and $6637 \mu \mathrm{g} / \mathrm{g}$. ${ }^{31}$ In particular, different varieties of strawberry showed concentrations between 539 and $1632 \mu \mathrm{g} / \mathrm{g}$, also applying the phloroglucinolysis method. ${ }^{14}$ 
Table 3. Proanthocyanidin Content after Phloroglucinolysis in Different Parts of Pomegranate Samples $(\mu \mathrm{g} / \mathrm{g}$ of FW), Pressed Aril Juice, and Commercial Juice $(\mathrm{mg} / \mathrm{L})^{a}$

\begin{tabular}{|c|c|c|c|c|c|c|}
\hline & husk & membrane & seed & $\operatorname{aril}^{b}$ & pressed aril juice $^{c}$ & commercial juice $^{c}$ \\
\hline (-)-gallocatechin & $732.1 \pm 130.5$ & $21.3 \pm 7.4$ & $43.2 \pm 7.1$ & $3.8 \pm 1.9$ & $2.1 \pm 0.7$ & $32.9 \pm 8.1$ \\
\hline (-)-gallocatechin adduct & $211.2 \pm 18.3$ & & & $2.2 \pm 0.4$ & $2.6 \pm 0.4$ & $12.8 \pm 2.0$ \\
\hline total & $943.3 \pm 120.2$ & $21.3 \pm 7.4$ & $43.2 \pm 7.1$ & $6.0 \pm 1.6$ & $4.7 \pm 1.1$ & $45.7 \pm 9.8$ \\
\hline (-)-epigallocatechin & $80.1 \pm 8.7$ & & & $1.9 \pm 0.3$ & $3.0 \pm 0.4$ & $12.2 \pm 1.2$ \\
\hline (-)-epigallocatechin adducts & $178.6 \pm 35.3$ & & & $1.4 \pm 0.2$ & $1.6 \pm 0.3$ & $6.3 \pm 0.6$ \\
\hline total & $258.7 \pm 37.7$ & & & $3.3 \pm 0.5$ & $4.6 \pm 0.6$ & $18.5 \pm 1.7$ \\
\hline$(+)$-catechin & $279.0 \pm 43.1$ & $22.3 \pm 3.8$ & $168.4 \pm 33.1$ & $12.1 \pm 2.8$ & $2.2 \pm 0.5$ & $19.7 \pm 2.5$ \\
\hline$(+)$-catechin adduct & $12.5 \pm 3.2$ & & $5.8 \pm 1.3$ & $1.0 \pm 0.1$ & $1.1 \pm 0.2$ & $4.5 \pm 1.6$ \\
\hline total & $291.5 \pm 46.1$ & $22.3 \pm 3.8$ & $174.2 \pm 33.6$ & $13.1 \pm 2.8$ & $3.3 \pm 0.6$ & $24.2 \pm 4.0$ \\
\hline (-)-epigallocatechin gallate & $121.9 \pm 17.7$ & & & $0.2 \pm 0.1$ & $0.4 \pm 0.2$ & $0.1 \pm 0.0$ \\
\hline (-)-epigallocatechin gallate adduct & $28.8 \pm 4.2$ & & & & & \\
\hline total & $150.7 \pm 21.3$ & & & $0.2 \pm 0.1$ & $0.4 \pm 0.2$ & $0.1 \pm 0.0$ \\
\hline (-)-epicatechin & $5.4 \pm 2.5$ & $2.0 \pm 0.8$ & $7.5 \pm 0.9$ & $2.4 \pm 0.4$ & $2.6 \pm 0.4$ & $6.1 \pm 1.0$ \\
\hline (-)-epicatechin adduct & $165.7 \pm 28.8$ & $5.3 \pm 1.5$ & $87.1 \pm 17.5$ & $8.7 \pm 0.6$ & $5.2 \pm 0.8$ & $50.8 \pm 16.0$ \\
\hline total & $171.1 \pm 31.3$ & $7.3 \pm 1.6$ & $94.6 \pm 16.8$ & $11.1 \pm 1.0$ & $7.8 \pm 1.1$ & $56.9 \pm 16.9$ \\
\hline$(+)$-epicatechin gallate & $9.1 \pm 1.7$ & & & $0.3 \pm 0.0$ & $0.4 \pm 0.0$ & $0.1 \pm 0.0$ \\
\hline (+)-epicatechin gallate adduct & $16.1 \pm 0.4$ & $0.5 \pm 0.1$ & $4.2 \pm 1.0$ & $0.4 \pm 0.1$ & $0.1 \pm 0.0$ & $1.4 \pm 0.4$ \\
\hline total & $25.2 \pm 1.9$ & $0.5 \pm 0.1$ & $4.2 \pm 1.0$ & $0.7 \pm 0.2$ & $0.5 \pm 0.1$ & $1.5 \pm 0.4$ \\
\hline total proanthocyanidins & $1840.5 \pm 160.6$ & $51.4 \pm 5.8$ & $316.2 \pm 51.0$ & $34.4 \pm 3.0$ & $21.3 \pm 3.1$ & $146.9 \pm 31.7$ \\
\hline $\mathrm{mDP}$ & $1.5 \pm 0.1$ & $1.2 \pm 0.1$ & $1.5 \pm 0.1$ & $1.9 \pm 0.1$ & $2.1 \pm 0.1$ & $1.8 \pm 0.1$ \\
\hline
\end{tabular}

${ }^{a}$ The results are the mean \pm standard deviation $(\mathrm{SD})$ of the data obtained in four pieces of pomegranate fruit $(n=4) .{ }^{b}$ Aril concentrations was calculated as the sum of $30 \%$ of the concentration of the residue obtained after juice extraction (press cake) and $70 \%$ of the concentration of the pressed aril juice. ${ }^{c}$ Juice concentration (pressed arils and commercial) was calculated as the sum of juice and pellet.

A)

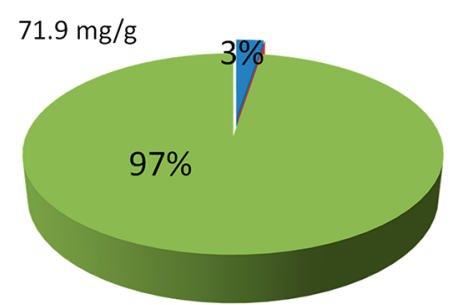

C) $4.4 \mathrm{mg} / \mathrm{g}$

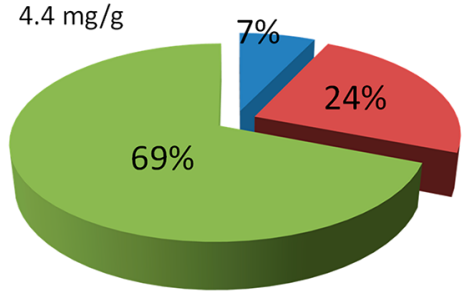

E) $2.5 \mathrm{~g} / \mathrm{L}$

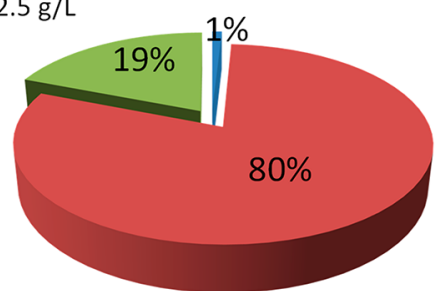

B)

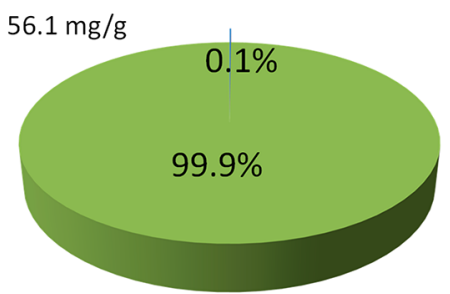

D) $2.7 \mathrm{mg} / \mathrm{g}$

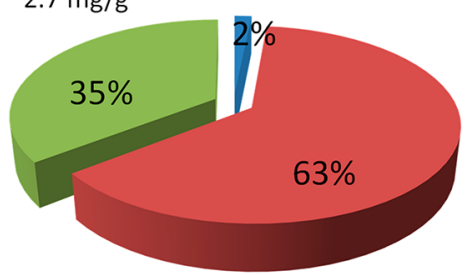

F) $2.2 \mathrm{~g} / \mathrm{L}$

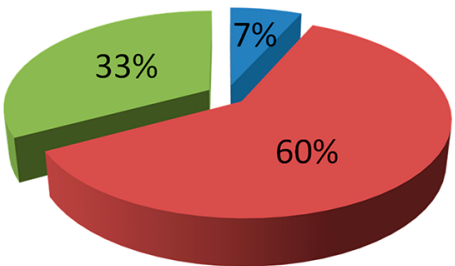

Figure 6. Total amount of phenolic compounds and percentages of proanthocyanidins (blue), anthocyanins (red), and ellagitannins (green) in different pomegranate samples: (A) husk, (B) membrane, (C) seeds, (D) arils, (E) pressed aril juice, and (F) commercial juice.

Pomegranate arils showed amounts similar to peach $(29 \mu \mathrm{g} /$ $\mathrm{g})$, kiwis $(37 \mu \mathrm{g} / \mathrm{g})$, or bananas $(40 \mu \mathrm{g} / \mathrm{g})$, fruit with the lowest amount of PAs among all of the fruit and beverages analyzed. ${ }^{26}$ With regard to beverages, the amount found in commercial pomegranate was similar to that found in milk chocolate (192 $\mathrm{mg} / \mathrm{L})$, cranberry juice (231 $\mathrm{mg} / \mathrm{L})$, Quercus leaf infusion
$(25.3-347 \mathrm{mg} / \mathrm{L}),{ }^{24}$ or red wine $(76.93-133.18 \mathrm{mg} / \mathrm{L})^{25}$ but much lower than that found in grape juice $(524 \mathrm{mg} / \mathrm{L})$ or green tea $(4572 \mathrm{mg} / \mathrm{L}) .^{23}$

The mDP values ranged from 1.2 to 2.1 , showing that samples had basically monomers and dimers, with a low amount of oligomers. These results are in agreement with those previously 
found for different varieties of pomegranate juice, where the $\mathrm{mDP}$ ranged from 1.0 to $3.2 .^{27}$ PAs with a low degree of polymerization are much likely to be absorbed through the gut barrier. ${ }^{32}$ In previous studies, absorption of dimeric PAs was observed in rats and humans, ${ }^{33,34}$ and even trimeric PCs were detected in rat urine samples. ${ }^{33}$ PAs with a higher degree of polymerization are not absorbed and reach the colon, where they are metabolized by the gut microbiota.

Comparison to the Ellagitannin and Anthocyanin Contents. The amount of PAs was compared to those obtained for ellagitannins (Supplementary Tables 1 and 2 of the Supporting Information) and anthocyanins (Supplementary Table 3 of the Supporting Information), the main compounds previously identified in pomegranate samples. ${ }^{35}$ Figure 6 summarizes the total phenolic content of the analyzed samples and the percentages of each group of compounds. The largest amount of polyphenols was observed in the husk $(71.9 \mathrm{mg} / \mathrm{g}$ ) and membranes $(56.1 \mathrm{mg} / \mathrm{g})$. Their phenolic contents were dominated by ellagitannins (97 and $99.9 \%$, respectively), whereas PAs represented only 3 and $0.1 \%$, respectively. Ellagitannins were also the main compounds in pomegranate seeds (69\%) but also contained $24 \%$ anthocyanins and $7 \%$ PAs. The lowest phenolic content $(2.7 \mathrm{mg} / \mathrm{g})$ was observed in the edible part, where PAs accounted for $2 \%$. Ellagitannins were again the predominant phenolics (63\%), although anthocyanins (35\%) were also detected. A different pattern was observed in juices, where anthocyanins were the most important constituents, representing $80 \%$ in pressed aril juice and $60 \%$ in commercial juice. PAs represented low percentages: $7 \%$ in commercial juice and only $1 \%$ in pressed aril juice.

As a conclusion, this report shows that PAs are present at significant amounts in the edible parts of pomegranate and are particularly relevant in commercial juices, both from the Mollar cultivar. Future studies should be considered to analyze and compare the content of PAs in fruit and juices from other pomegranate cultivars. PAs complement the large amounts of ellagitannins and anthocyanins present in pomegranates and should also be considered as contributors to the health effects of pomegranate juice, particularly because they are mainly flavan 3ol monomers and dimers that show higher bioavailability than larger oligomers and polymers.

\section{ASSOCIATED CONTENT}

\section{S Supporting Information}

The Supporting Information is available free of charge on the ACS Publications website at DOI: 10.1021/acs.jafc.8b07155.

Quantitation of ellagitannins obtained after acid hydrolysis in different parts of pomegranate, pressed aril juice, and commercial juice (Supplementary Table 1), concentration of the main ellagitannins and ellagic acid derivatives present in different parts of pomegranate, homemade juice, and commercial juice (Supplementary Table 2), and concentration of anthocyanins in different parts of pomegranate, pressed aril juice, and commercial juice (Supplementary Table 3) (PDF)

\section{AUTHOR INFORMATION}

\section{Corresponding Authors}

*Telephone: +34-968-396200, ext. 6254. Fax: +34968-396213.

E-mail: fatomas@cebas.csic.es.

*E-mail: rgvillalba@cebas.csic.es.

\section{ORCID}

Francisco A. Tomás-Barberán: 0000-0002-0790-1739

\section{Funding}

This research was funded by CSIC 201870E014, Fundación Séneca de la Región de Murcia, Ayudas a Grupos de Excelencia 19900/GERM/15, RIS-3 BEVETECH, and AGL2015-73107EXP/AEI.

\section{Notes}

The authors declare no competing financial interest.

\section{ACKNOWLEDGMENTS}

The authors are grateful to AMC INNOVA (Murcia, Spain) for supplying the pomegranate juice samples.

\section{ABBREVIATIONS USED}

PA, proanthocyanidin; C, catechin; EC, epicatechin; GC, gallocatechin; EGC, epigallocatechin; ECG, epicatechin gallate; EGCG, epigallocatechin gallate

\section{REFERENCES}

(1) Faria, A.; Calhau, C. The bioactivity of pomegranate: Impact on health and disease. Crit. Rev. Food Sci. Nutr. 2011, 51 (7), 626-634.

(2) Asgary, S.; Keshvari, M.; Sahebkar, A.; Sarrafzadegan, N. Pomegranate consumption and blood ptressure: A review. Curr. Pharm. Des. 2017, 23 (7), 1042-1050.

(3) Viuda-Martos, M.; Fernández-López, J.; Pérez-Álvarez, J. A. Pomegranate and its many functional components as related to human health: A review. Compr. Rev. Food Sci. Food Saf. 2010, 9 (6), 635-654.

(4) Gil, M. I.; Tomás-Barberán, F. A.; Hess-Pierce, B.; Holcroft, D. M.; Kader, A. A. Antioxidant activity of pomegranate juice and its relationship with phenolic composition and processing. J. Agric. Food Chem. 2000, 48 (10), 4581-4589.

(5) Sentandreu, E.; Cerdán-Calero, M.; Sendra, J. M. Phenolic profile characterization of pomegranate (Punica granatum) juice by highperformance liquid chromatography with diode array detection coupled to an electrospray ion trap mass analyzer. J. Food Compos. Anal. 2013, 30 (1), 32-40.

(6) Fischer, U. A.; Carle, R.; Kammerer, D. R. Identification and quantification of phenolic compounds from pomegranate (Punica granatum L.) peel, mesocarp, aril and differently produced juices by HPLC-DAD-ESI/MS ${ }^{n}$. Food Chem. 2011, 127 (2), 807-821.

(7) Borges, G.; Mullen, W.; Crozier, A. Comparison of the polyphenolic composition and antioxidant activity of European commercial fruit juices. Food Funct. 2010, 1, 73-83.

(8) Sentandreu, E.; Navarro, J. L.; Sendra, J. M. LC-DAD-ESI/MS ${ }^{n}$ determination of direct condensation flavanol-anthocyanin adducts in pressure extracted pomegranate (Punica granatum L.) juice. J. Agric. Food Chem. 2010, 58 (19), 10560-10567.

(9) Hellström, J. K.; Törrönen, A. R.; Mattila, P. H. Proanthocyanidins in common food products of plant origin. J. Agric. Food Chem. 2009, 57 (17), 7899-7906.

(10) Macáková, K.; Kolečkář, V.; Cahlíková, L.; Chlebek, J.; Hošt'álková, A.; Kuča, K.; Jun, D.; Opletal, L. Tannins and Their Influence on Health. Recent Advances in Medicinal Chemistry; Elsevier: Amsterdam, Netherlands, 2014; Vol. 1, Chapter 6, pp 159-208, DOI: 10.1016/B978-0-12-803961-8.50006-3.

(11) Koleckar, V.; Kubikova, K.; Rehakova, Z.; Kuca, K.; Jun, D.; Jahodar, L.; Opletal, L. Condensed and hydrolysable tannins as antioxidants influencing the health. Mini-Rev. Med. Chem. 2008, 8 (5), 436-447.

(12) Gu, L. Analysis methods of proanthocyanidins. In Analysis of Antioxidant-Rich Phytochemicals; Xu, Z., Howard, L. R., Eds.; WileyBlackwell: Oxford, U.K., 2012; Chapter 8, pp 247-274, DOI: 10.1002/ 9781118229378.ch8. 
(13) Kennedy, J. A.; Jones, G. P. Analysis of proanthocyanidin cleavage products following acid-catalysis in the presence of excess phloroglucinol. J. Agric. Food Chem. 2001, 49 (4), 1740-1746.

(14) Buendía, B.; Gil, M. I.; Tudela, J. A.; Gady, A. L.; Medina, J. J.; Soria, C.; López, J. M.; Tomás-Barberán, F. A. HPLC-MS analysis of proanthocyanidin oligomers and other phenolics in 15 strawberry cultivars. J. Agric. Food Chem. 2010, 58 (7), 3916-3926.

(15) Gris, E. F.; Mattivi, F.; Ferreira, E. A.; Vrhovsek, U.; Pedrosa, R. C.; Bordignon-Luiz, M. T. Proanthocyanidin profile and antioxidant capacity of Brazilian Vitis vinifera red wines. Food Chem. 2011, 126 (1), 213-220.

(16) Jin, A.; Ozga, J. A.; Lopes-Lutz, D.; Schieber, A.; Reinecke, D. M. Characterization of proanthocyanidinds in pea (Pisum sativum L.), lentil (Lens culinaris L.), and faba bean (Vicia faba L.) seeds. Food Res. Int. 2012, 46 (2), 528-535.

(17) Wissam, Z.; Ghada, B.; Wassim, A.; Warid, K. Effective extraction of polyphenols and proanthocyanidins from pomegranate's peel. Int. J. Pharm. Pharm. Sci. 2012, 4, 675-682.

(18) de Pascual-Teresa, S.; Santos-Buelga, C.; Rivas-Gonzalo, J. C. Quantitative analysis of flavan-3-ols in Spanish foodstuffs and beverages. J. Agric. Food Chem. 2000, 48 (11), 5331-5337.

(19) Smrke, S.; Vovk, I. Comprehensive thin-layer chromatography mass spectrometry of flavanols from Juniperus communis L. and Punica granatum L. J. Chrom. A 2013, 1289, 119-126.

(20) Plumb, G. W.; De Pascual-Teresa, S.; Santos-Buelga, C.; RivasGonzalo, J. C.; Williamson, G. Antioxidant properties of gallocatechin and prodelphinidins from pomegranate peel. Redox Rep. 2002, 7 (1), 41-46.

(21) Jakobek, L.; García-Villalba, R.; Tomás-Barberán, F. A. Polyphenolic characterisation of old local apple varieties from Southeastern European region. J. Food Compos. Anal. 2013, 31 (2), 199-211.

(22) García-Villalba, R.; Espín, J. C.; Aaby, K.; Alasalvar, C.; Heinonen, M.; Jacobs, G.; Voorspoels, S.; Koivumäki, T.; Kroon, P. A.; Pelvan, E.; Saha, S.; Tomás-Barberán, F. A. Validated method for the characterization and quantification of extractable and nonextractable ellagitannins after acid hydrolysis in pomegranate fruits, juices and extracts. J. Agric. Food Chem. 2015, 63 (29), 6555-6566.

(23) Del Río, D.; Stewart, A. J.; Mullen, W.; Burns, J.; Lean, M. E. J.; Brighenti, F.; Crozier, A. HPLC-MS ${ }^{n}$ analysis of phenolic compounds and purine alkaloids in green and black tea. J. Agric. Food Chem. 2004, 52 (10), 2807-2815.

(24) García-Villalba, R.; Espín, J. C.; Tomás-Barberán, F. A.; RochaGuzmán, N. E. Comprehensive characterization by LC-DAD-MS/ MS of the phenolic composition of seven Quercus leaf teas. J. Food Compos. Anal. 2017, 63, 38-46.

(25) Monagas, M.; Gómez-Cordovés, C.; Bartolomé, B.; Laureano, O.; Ricardo da Silva, J. M. Monomeric, oligomeric, and polymeric falvan-3-ol composition of wines and grapes from Vitis vinifera L. Cv. Graciano, Tempranillo, and Cabernet Sauvignon. J. Agric. Food Chem. 2003, 51, 6475-6481.

(26) Gu, L.; Kelm, M. A.; Hammerstone, J. F.; Beecher, G.; Holden, J.; Haytowitz, D.; Gebhardt, S.; Prior, R. L. Concentrations of proanthocyanidins in common foods and estimations of normal consumption. J. Nutr. 2004, 134 (3), 613-617.

(27) Türkyilmaz, M.; Özkan, M. Effects of condensed tannins on anthocyanins and colour of authentic pomegranate (Punica granatum L.) juices. Food Chem. 2014, 164, 324-331.

(28) Borges, G.; Crozier, A. HPLC-PDA-MS fingerprinting to assess the authenticity of pomegranate beverages. Food Chem. 2012, $135,1863-1867$.

(29) Hammerstone, J. F.; Lazarus, S. A.; Schmitz, H. H. Procyanidin content and variation in some commonly consumed foods. J. Nutr. 2000, 130, 2086S-2092S.

(30) Hernández-Jiménez, A.; Gómez-Plaza, E.; Martínez-Cutillas, A.; Kennedy, J. A. Grape skin and seed proanthocyanidins from Monastrell x Syrah grapes. J. Agric. Food Chem. 2009, 57, 10798-10803.

(31) Wu, X.; Gu, L.; Prior, R. L.; McKay, S. Characterization of anthocyanins and proanthocyanidins in some cultivars of Ribes, Aronia, and Sambucus and their antioxidant capacity. J. Agric. Food Chem. 2004, 52 (26), 7846-7856.

(32) Zhang, L.; Wang, Y.; Li, D.; Ho, C. T.; Li, J.; Wan, X. The absorption, distribution, metabolism and excretion of procyanidins. Food Funct. 2016, 7, 1273-1281.

(33) Serra, A.; Macia, A.; Romero, M. P.; Valls, J.; Blade, C.; Arola, L.; Motilva, M. J. Bioavailability of procyanidin dimers and trimers and matrix food effects in in vitro and in vivo models. Br. J. Nutr. 2010, 103, 944-952.

(34) Sano, A.; Yamakoshi, J.; Tokutake, S.; Tobe, K.; Kubota, Y.; Kikuchi, M. Procyanidin B1 is detected in human serum after intake of proanthocyanidin-rich grape seed extract. Biosci., Biotechnol., Biochem. 2003, 67, 1140-1143.

(35) Ambigaipalan, P.; de Camargo, A. C.; Shahidi, F. Phenolic compounds of pomegranate byproducts (outer skin, mesocarp, divider membrane) and their antioxidant activities. J. Agric. Food Chem. 2016, $64,6584-6604$. 